\title{
Drop shape analysis for determination of dynamic contact angles by double sided elliptical fitting method
}

\section{Andersen, Nis Korsgaard; Taboryski, Rafael J.}

Published in:

Measurement Science and Technology

Link to article, DOI:

10.1088/1361-6501/aa5dcf

Publication date:

2017

Document Version

Peer reviewed version

Link back to DTU Orbit

Citation (APA):

Andersen, N. K., \& Taboryski, R. J. (2017). Drop shape analysis for determination of dynamic contact angles by double sided elliptical fitting method. Measurement Science and Technology, 28, [047003]. https://doi.org/10.1088/1361-6501/aa5dcf

\section{General rights}

Copyright and moral rights for the publications made accessible in the public portal are retained by the authors and/or other copyright owners and it is a condition of accessing publications that users recognise and abide by the legal requirements associated with these rights.

- Users may download and print one copy of any publication from the public portal for the purpose of private study or research.

- You may not further distribute the material or use it for any profit-making activity or commercial gain

- You may freely distribute the URL identifying the publication in the public portal 


\title{
Drop shape analysis for determination of dynamic contact angles by double sided elliptical fitting method
} Nis Korsgaard Andersen and Rafael Taboryski*

Department of Micro- and Nanotechnology, Technical University of Denmark, 2800 Kongens Lyngby, Denmark

* rata@nanotech.dtu.dk

\begin{abstract}
Contact angle measurements is a fast and simple way to measure surface properties and is therefore widely used to measure surface energy and quantify wetting of a solid surface by a liquid substance. In common praxis contact angle measurements are done with sessile drops on a horizontal surface fitted to a drop profile derived from the Young-Laplace equation. When measuring the wetting behaviour by tilting experiments this is not possible since it involves moving drops that are not in equilibrium. Here we present a fitting technique capable of determining the contact angle of asymmetric drops with very high accuracy even with blurry or noisy images. This we do by splitting the trace of a drop into a left and right part at the apex and then fit each side to an ellipse.
\end{abstract}

\section{Keywords}

Contact angle, wetting behaviour, roll-off angle, drop shape analysis, axisymmetric drop shape analysis (ADSA), triple-line, and contact angle hysteresis. 
Measurements of contact angles between liquids and solids are widely used to determine properties of either the liquid or the solid. [1] When measuring the contact angle it is only the chemical properties of a few of the outermost atomic layers in the solid that affects the liquid, this makes contact angles a very simple way to measure surface properties. [2] The simplest and most used method to measure the contact angles of a drop is by depositing a drop of liquid on a solid surface and acquire a digital image of the drop in profile. [3] The image is then analysed to extract the coordinates for the drop profile and determine the position of the solid-liquid interface. To extract the contact angle from the obtained data the drop profile is fitted to an equation, which is evaluated at the triple-line. For a drop sitting on a horizontal and homogeneous surface, we can assume that the drop is axisymmetric around the vertical axis and the drop shape is therefore completely described by the hydrostatic Young Laplace equation. Fitting to the Young Laplace equation is called axisymmetric drop shape analysis (ADSA) and was at first limited to drops where the apex of the drop was visible[4] and have later been improved to be able to fit drops with only parts of the drop shape being visible (ADSA-No Apex). [5] It is widely accepted within the field of measuring contact angles that fitting to the Young Laplace equation provides measurements with the highest possible accuracy and improvements to the technique only concerns the fitting algorithm, the determination of the baseline and the exact position of the drop perimeter. An example of a different approach to fit the acquired image to the Young Laplace equation is Theoretical Image Fitting Analysis (TIFA) [4, 6] where theoretical images are generated and compared to the acquired image, thereby circumventing the need for an edge detection algorithm. The validity of the Young Laplace equation is however limited to symmetric drop shapes. This means that for measurement of the dynamic contact angles by tilting experiments there is a need for a different equation to fit the drop perimeter to. Several examples of this are derived approximations to the Young Laplace equation assuming some out of plane shape of the drop[7] or purely arbitrary equations like, cubic splines[8] or polynomials.[9, 10]

When choosing fitting algorithm there are two important properties that should be considered; firstly, the amount of data-points that can be fitted to the equation. This usually involves both a minimum number of points to achieve the desired precision and a maximum number of points where the equation is a good approximation to the drop shape. Secondly, the ability to extrapolate the drop shape outside the region of fitted data points, since optical distortions at the triple-point require the fitted equation to be extrapolated down to the baseline. 
For the axisymmetric case, the Young-Laplace based fitting methods are able to use the whole perimeter of the drop while being very accurate at extrapolating the drop shape since it is derived from the physical properties of the drop. For tilted drops, the most commonly used method is polynomial fitting due to its simplicity. According to Weierstrass approximation theorem polynomials can be as good a fit to a continuous function on a closed interval as desired, [11] this means that polynomials always will be able to fit parts of the drop shape, even for oddly shaped drops impacting a surface or under influence of electric fields etc. Polynomials are usually used as interpolants, where higher order or piecewise polynomials in general can be used to get results that are more accurate. For fitting drop shapes and measuring contact angles, the polynomial will however be used to extrapolate the drop shape making the degree of polynomial a trade-off between the maximum amount of points that can be used for the fit and the accuracy on the extrapolated drop shape. This makes polynomial fitting very sensitive to noise in the image and especially to blurry edges or optical defects at the contact point. In order to be able to fit to all data points on the drop perimeter we have found that fitting advancing and receding sides of the drop separately with two ellipses gives very accurate results for most real drops. This can be seen as a generalization of the concept presented by El Sherbini et.al. [7] where they fit a vertically inclined drop to two circular segments divided at the apex of the drop.

To be able to compare our elliptical fitting method to the more common polynomial fitting we have implemented a polynomial fitting algorithm together with the elliptical fitting method. In this way, we can ensure that the extracted drop perimeter and baseline detection is the same and that the difference in results only comes from the difference in fitting method. In our implementation of polynomial fitting we rotate and translate the data points for each side of the drop so that the curve of data points $(x, y)$ fulfil $\left.\frac{d y}{d x}\right|_{x=0}=0$ and $\langle x\rangle=0$. In this way we have good conditions for the polynomial fit regardless of the contact angle of the drop and thereby circumvent the difficulty in fitting polynomials to nearly vertical profiles for drops with a contact angle close to $90^{\circ}$. For implementation of polynomial fitting, it is required to select the degree of the polynomial and the amount of points used in the fit. We choose this by simulating points on circular segments with a small scatter and fitting the points to polynomials of various degrees. We found that fourth order polynomials are good for extrapolation of the slope while still being able to fit a large arc of the segment with high accuracy. These simulations are explained in detail in supplementary materials.

The drop shape analysis has been implemented in MATLAB (R2016a) and can be broken down into a series of steps, each shown in Figure 1. Step a), extract the perimeter of the drop. This is done using the algorithm 
and script presented by Trujillo-Pino et al.. [12] The method of Trujillo-Pino et.al. provides edge detection with subpixel accuracy that is very similar to those obtained by sigmoidal fitting[10] of the edges while being computationally faster and more accurate at points where edges are close to each other, e.g. at the triple-line for very high or very low contact angles. Step b), determine the baseline by finding the reflection and then calculating the intersection between linear fits made to the data points just above and below the reflection. This should preferably be done on several recorded frames and then averaged to obtain a precise positioning of the baseline. Step c), if using polynomial fit, we need to select the amount of points needed for the fit. For this, we use a geometric relation for circular drops, that the arc between apex and contact point equals the contact angle. To select $s$ out of $n$ data points on the perimeter of the drop corresponding to an $\operatorname{arc} \alpha$ with a drop contact angle $C A$, we use the relation $s=n \cdot \frac{\alpha}{C A}$. If the selected arc is smaller than the contact angle, all data points from apex to triple-line are used. For all polynomial fits presented in this paper we use data points in an arc of $\alpha=60^{\circ}$, see supplementary material for details. Step d) fit the obtained data to the function of choice. We show both polynomial and elliptic fits. Polynomials are fitted using standard linear least squares fitting whereas ellipses are fitted using the direct elliptic fitting method proposed by Fitzgibbon et al..[13] Step e), evaluate the slope of the fitted function at the intersection between fit and baseline. From this, the contact angle is calculated.

(a)

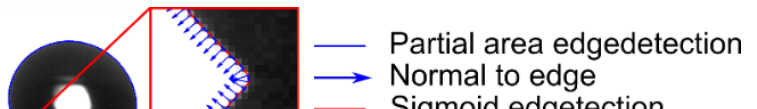

(b)

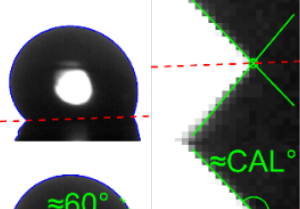

Sigmoid edgetection

(c)

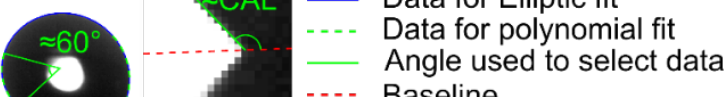

(d)
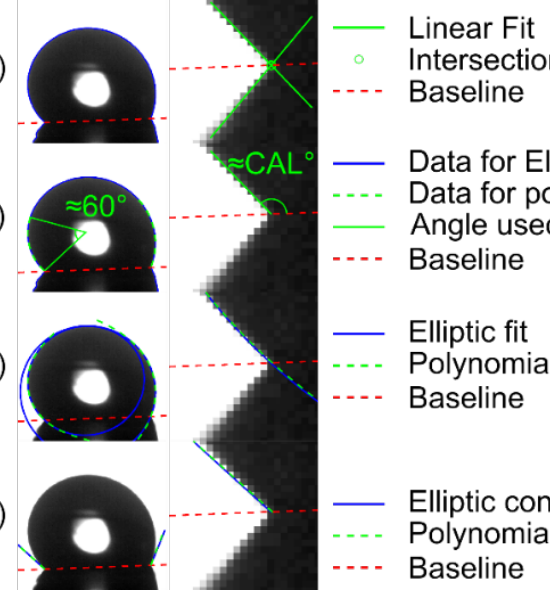

Figure 1 Step-by-step implementation of the contact angle fitting algorithm. (a) Edge detection. (b) Baseline detection. (c) Selection of data points used in polynomial fit. (d) Elliptic and polynomial fit. (e) Evaluation of fit at the intersection with baseline to obtain the contact angle. 
To evaluate the implementation of both the double-sided elliptic and the polynomial fitting algorithm we have constructed a series of synthetic images. We constructed all synthetic images to resemble images obtained by our goniometer (Attension theta, Biolin Scientific) but with well-defined contact angles. After validating the numeric implementation of the fitting algorithms, we use the generation of synthetic images to determine the sensitivity of the algorithms to different kinds of distortions. Each synthetic image is generated by plotting circular segments with a smooth transition from black (drop) to white (open space). This is implemented by calculating the position of centre of the circular segment corresponding to a specific contact angle, in Figure 2 we have sketched a synthetic drop where the centre of the circular segment is positioned in $(0, D)$ where $D=-R \cos C A$, the radius $R$ is chosen so the drop can fit into the image. The drop edge is generated by evaluating a cumulative distribution function of a Gaussian distribution $F(s)=\frac{1}{2}\left[1+\operatorname{erf}\left(\frac{s-R}{\sigma \sqrt{2}}\right)\right]$ where $s$ is the distance to $C, \mathrm{R}$ is the radius of the drop and $\sigma$ is the standard deviation describing the width of the greyscale transition. By analysing the goniometer images presented in this paper we find that the average width of the transition is $\sigma=0.85 \pm 0.19$ pixel, and unless otherwise stated we have used $\sigma=0.85$ pixel to generate the synthetic images.

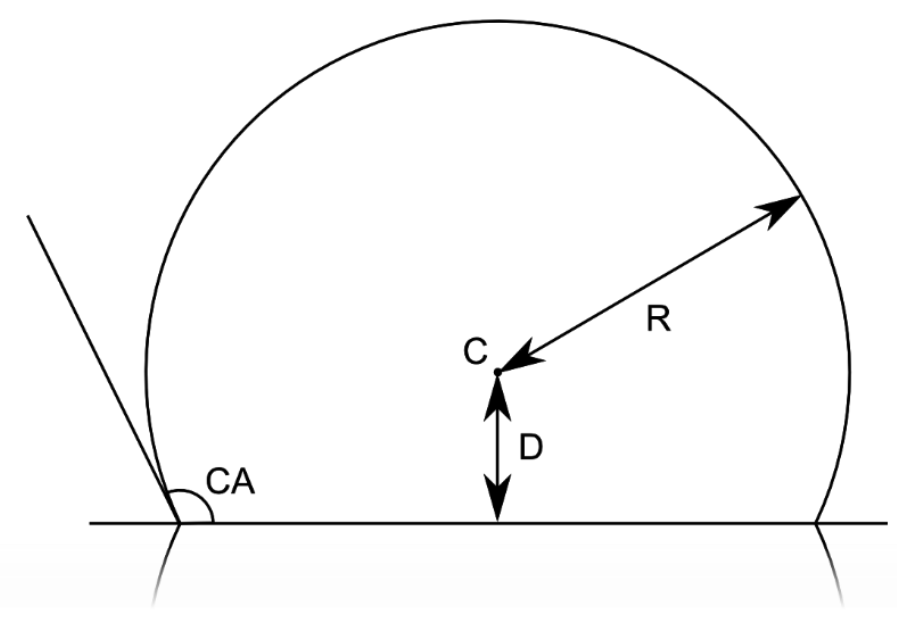

Figure 2 Schematic drawing of the geometry used to generate synthetic images.

By generating synthetic images with contact angles ranging from $10^{\circ}$ to $170^{\circ}$ and fitting the images with both polynomial- fitting and elliptical fitting we obtain the data presented in Figure 3. From this, we see that the rotation of the drop boundary for polynomial fits ensures high accuracy also for contact angles near $90^{\circ}$. When comparing the performance of our implementation of polynomial fit with the results presented by Chini et al. [10] we see slightly worse performance for our program for the synthetic image resolution used; this can be improved by generating synthetic images with higher resolution (see the 
Supplementary Material). Instead, we choose to evaluate the accuracy of our fitting technique by generating as realistic drop images as possible in bitmaps with a resolution of $512 \times 337$ pixels and a black to white transition width of 0.85 pixels. For almost all contact angles, the elliptic fit determines the contact angle more accurate that the polynomial fit, this is to be expected since the synthetic drops are generated as circular segments that are fitted perfectly by ellipses. The seemingly stochastic variation of the error on the determined contact angle is directly related to the chosen resolution of the synthetic image. When generating the synthetic images there will be a loss of information due to digitizing of the geometric shape into pixels. The error due to this information loss is directly linked to the exact digitizing of the drop and will therefore be different for different resolutions (see the Supplementary Material).

In the generation of synthetic images, it is directly possible to vary the sharpness of drop edges and add noise to the generated image. By changing the width of the greyscale transition in the image generation, we evaluate the error introduced by blurry images, for instance if the drop profile is taken slightly out of focus. We have varied the width of the greyscale transition from 0 pixels (completely sharp) to 3 pixels (blurry to the naked eye) of a drop with a contact angle of $140^{\circ}$ and presented the error in contact angle in Figure $3 b)$. For most synthetic drop shapes in the parameter space investigated, except for very low contact angles, where the elliptic fits are very sensitive to choice of the drop centre, and very noisy images, we see that the elliptic contact angle measurement is more accurate than the polynomial fit. It is, however, also apparent that the two graphs follow the same trend (on a log scale) indicating that the main error arises from the profile extraction that is the same for both drops. The minimum error for both fitting methods around $\sigma=0.5$ is a consequence of the edge detection algorithm that utilizes the greyscale values in a black-to-white transition to determine the true edge. When digitizing the drop geometry using $\sigma=0$ there resulting image is purely black and white and the edge detection will be more inaccurate compared to images with a narrow greyscale transition. For $\sigma=0.5$ the edge detection algorithm returns sub-pixel locations that are very close to the real drop perimeter, thereby resulting in a very low fitting error.

All synthetic drops presented until now had realistic blurry edges but were otherwise noise free. In order to verify that our algorithm is able to produce correct results when including the noise from real images we have added Gaussian noise to the synthetic images. This is done by adding/subtracting Gaussian distributed numbers to all pixel values where the mean of the Gaussian is zero and the standard deviation is $\mu$ and pixel values range from 0 (black) to 1 (white). We have increased the standard deviation of the Gaussian noise from $\mu=0$ (noise free) to $\mu=0.12$ (noisy) and plotted the resulting error on the contact angle in Figure $3 c)$. 

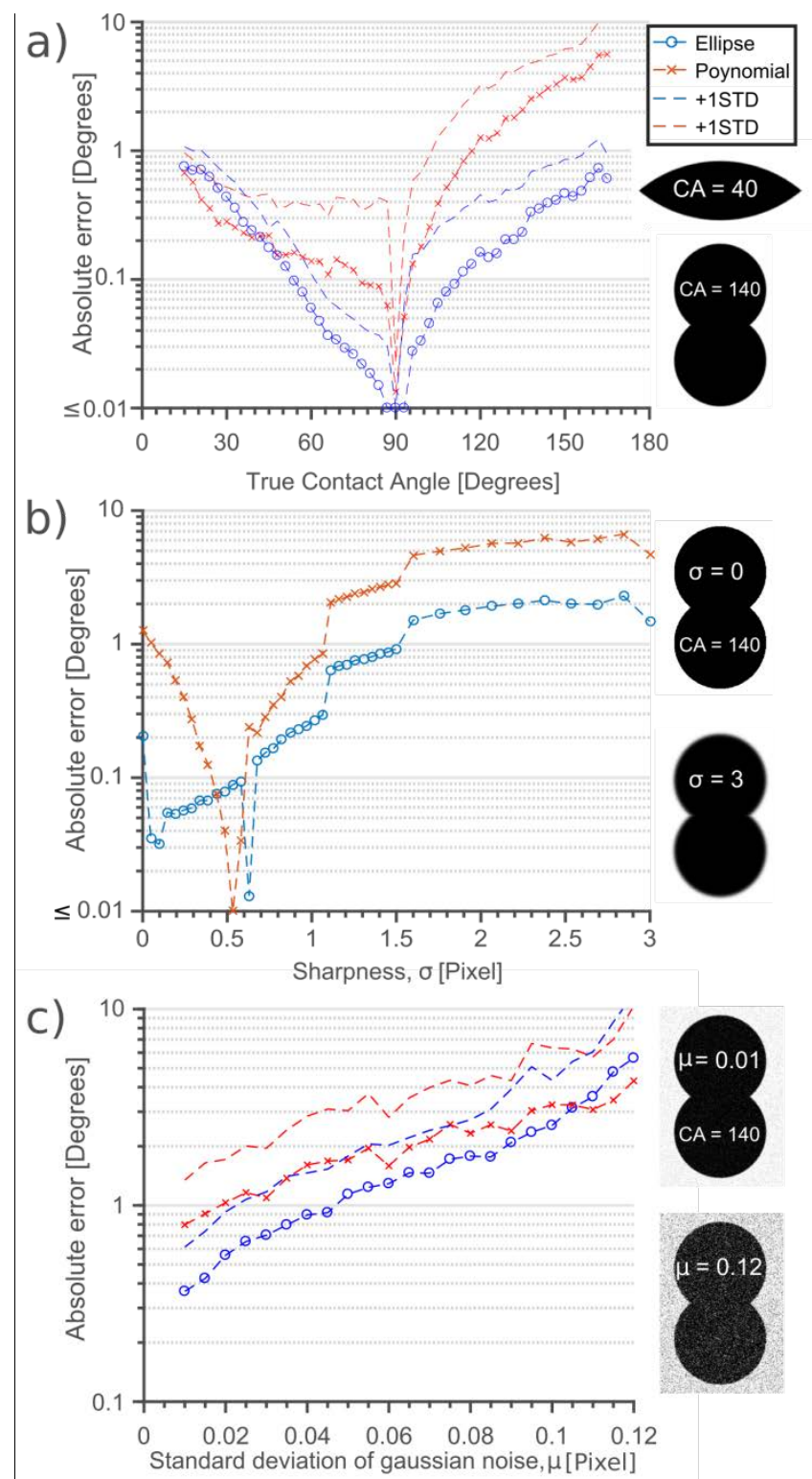

Figure 3 Evaluation of absolute error on the contact angle estimation using synthetic images. (a) The absolute error in contact angle vs. the true contact angle from $10^{\circ}$ to $170^{\circ}$. The curves results from fitting 500 drops with a one pixel gaussian distributed position of the drop centres. (b) The effect of varying the width of the black to white transition at the drop edge, simulating blurry images. (c) The effect of adding noise to the synthetic images.

In order to evaluate the performance of real images of drops we have captured drop profiles of seven sample drops shown in Figure 4. The structured surfaces for drops (3), (4), (6), and (7) in Figure 4., are: (3) randomly structured PP substrate, [14] (4) FDTS coated Si substrate fabricated by same method as in Søgard et al. [15] but with hierarchical pillar structures with $2 \%$ resulting surface coverage, (6) same 
substrate as (3), and (7) same substrate as (4). These images are chosen to test a wide range of contact angles and some have been tilted in order to measure contact angle hysteresis. To get the range in contact angles the liquid and solid have been combined in the following way: 1) oleic acid on flat polypropylene, 2) water on flat $A B S, 3$ ) water on micro structured polypropylene, 4) water on micro and nano structured silicon surface coated with perfluorodecyltrichlorosilane (FDTS), 5-7) same as 2-4), tilted until onset of movement by $49.5^{\circ}, 32.8^{\circ}$ and $8.3^{\circ}$ respectively. The drop in image 1 , figure 4 , does not start moving for any tilting value and is therefore not shown. For most of the drop images, there is excellent agreement between the contact angles calculated by elliptic and polynomial fitting, only for the drop on the micro and nano structured silicon surface there are significant deviations. For micro structured surfaces where the drop is resting on top of the asperities in the so called Cassie-Baxter state it has been shown that the real advancing contact angle is $180^{\circ}$ due to the physical transition between the tops of asperities.[16]lt is per se not problematic that the fitting algorithms undershoots this value since we know the true value but it shows the difficulties in determining very high contact angles. [17]

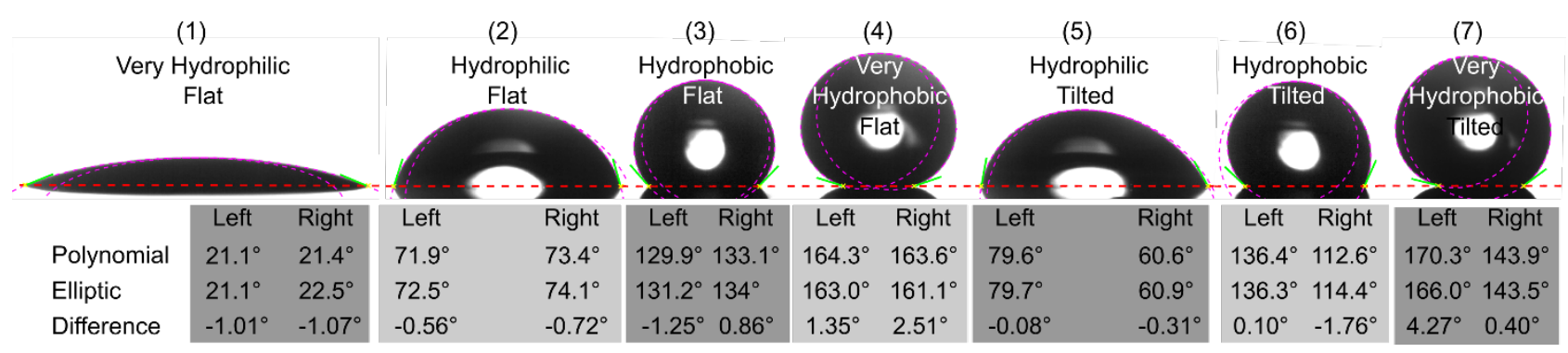

Figure 4 Calculated contact angles of 7 drops spanning from very low contact angles to very large, image 14 are resting on a horizontal surface while in image 5-7 the substrate have been tilted with the camera to obtain asymmetric drops. Except for contact angles above 160', there is good agreement between the elliptic and polynomial fitting method.

We demonstrate the strength of the elliptic fitting method by measuring the dynamic contact angles in a tilting experiment. In this experiment we place a drop on the surface and tilt surface and camera until the drop slides or rolls off. During the experiment, the drop shape is captured by the camera and saved with the corresponding tilt value.

In our experimental setup the sample is placed on an $x-y-z$ stage, which introduces slight mechanical instability, this makes the stage follow a slightly different trajectory than the camera. The different trajectory of stage and camera results in the image of the drop being shifted and rotated during the experiment. In order to subtract this mechanical shift we have recorded a calibration grid during a tilting 
experiment. By tracing the calibration grid while tilting, we can obtain the shift and rotation of the stage in the camera view as a function of tilt angle. When analysing the frames captured during the experiment, the drop profiles are first extracted and then transformed to the coordinate system of the first frame by shift and rotation. This enables us to average baseline positions from all frames to determine the baseline with high accuracy. By having all drop profiles transformed to the same coordinate system, it is also possible to get precise information on the movement of the triple-line.

In Figure 5 we have plotted data obtained for a tilting experiment using a micro- and nanostructured silicon surface coated with FDTS with a $10 \mu \mathrm{l}$ drop of $24 \%$ ethanol and $76 \%$ deionized water. The sample was tilted with $0.5 \%$ and captured with a framerate of 1 frame per second. With the information from the moving triple-line, we can see that the drop initially spreads by advancing on the left (downhill) side while being stuck on the right (uphill) side. At $10.8^{\circ}$ tilt the right triple-line starts moving and the contact angles in this frame are taken as respectively the advancing and the receding contact angle. The slight negative movement of the right triple line just before the onset of movement is due to the optical distortions on the drop edge influencing the determination of the position of the triple-line. Without the information of the triple-line movement one could easily use the wrong frame resulting in erroneous roll-off and dynamic contact angles. After the onset of drop movement, it takes the drop several seconds with increasing inclination before the drop completely rolls off the surface. During this time there is a zipping like detachment process where the drop detaches individual pillars one a time.[18]

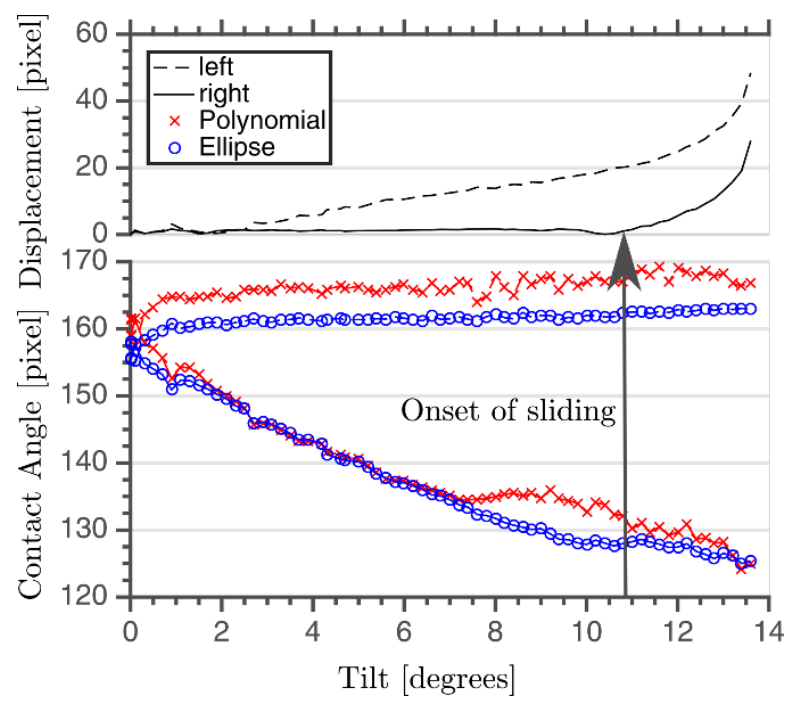

Figure 5 Displacement of the right and left triple line together with the contact angles measured by polynomial and elliptic fitting during tilt experiment. 
During the tilting of the sample, we measure the same contact angle on the left side and a decreasing contact angle on the right side. This confirms that the contact angle hysteresis and the roll-off angle of drops in the Cassie-Baxter state are solely governed by the receding contact angle.[15]

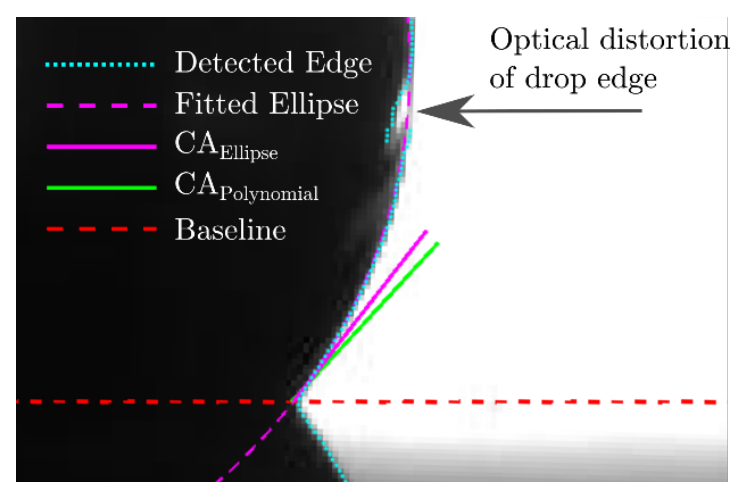

Figure 6 Fitting of the drop tilted $10^{\circ}$ from the sequence presented in figure 5

In the region just before drop movement starts, there is significant difference in the result between polynomial and elliptical fitting. The difference in determined contact angle arises due to lens effects in the drop producing white areas on the right side of the drop, especially close to the triple-line. An example of such optical distortion is shown in figure 6 . Since the polynomial fitting is more sensitive to optical distortions it produces significant error in the contact angle whereas elliptical fitting is much more stable and shows a smooth decrease of the contact angle as a function of tilting angle. Since the lens effects often occur just before the onset of movement, it is crucial to use a method with the stability of our elliptic fit to measure the correct contact angle.

In conclusion, we have presented a new method for fitting and measuring contact angles by the tilting method. This we have done by fitting ellipses to left and right sides of the drop profile. The double-sided elliptical fitting method has been compared to the well-known polynomial fitting, and the implementation of both algorithms has been validated using realistic synthetic images. By using double sided elliptical fitting it is possible to achieve much higher tolerance for optical distortions of the drop profile. Finally, we have shown that this is crucial in tilting experiments where lens effects in the drop distort the receding side of the drop profile, particularly around the triple-line. 
References

[1] Fowkes F M 1964 Contact Angle, Wettability, and Adhesion vol 43: AMERICAN CHEMICAL SOCIETY)

[2] Taherian F, Marcon V, van der Vegt N F A and Leroy F 2013 What Is the Contact Angle of Water on Graphene? Langmuir 29 1457-65

[3] Eral H B, t Mannetje D J C M and Oh J M 2013 Contact angle hysteresis: a review of fundamentals and applications Colloid and Polymer Science 291 247-60

[4] Rotenberg Y, Boruvka L and Neumann A W 1983 DETERMINATION OF SURFACE-TENSION AND CONTACT-ANGLE FROM THE SHAPES OF AXISYMMETRIC FLUID INTERFACES Journal of Colloid and Interface Science 93 169-83

[5] Kalantarian A, David R and Neumann A W 2009 Methodology for High Accuracy Contact Angle Measurement Langmuir 25 14146-54

[6] Cabezas M G, Bateni A, Montanero J M and Neumann A W 2004 A new drop-shape methodology for surface tension measurement Applied Surface Science 238 480-4

[7] ElSherbini A I and Jacobi A M 2004 Liquid drops on vertical and inclined surfaces II. A method for approximating drop shapes Journal of Colloid and Interface Science 273 566-75

[8] Stalder A F, Kulik G, Sage D, Barbieri L and Hoffmann P 2006 A snake-based approach to accurate determination of both contact points and contact angles Colloids and Surfaces a-Physicochemical and Engineering Aspects 286 92-103

[9] Atefi E, Mann J A, Jr. and Tavana H 2013 A Robust Polynomial Fitting Approach for Contact Angle Measurements Langmuir 29 5677-88

[10] Chini S F and Amirfazli A 2011 A method for measuring contact angle of asymmetric and symmetric drops Colloids and Surfaces a-Physicochemical and Engineering Aspects 388 29-37

[11] Jeffreys H and Swirles Jeffreys B 1999 Methods of Mathematical Physics: Cambridge University Presss)

[12] Trujillo-Pino A, Krissian K, Aleman-Flores M and Santana-Cedres D 2013 Accurate subpixel edge location based on partial area effect Image and Vision Computing 31 72-90

[13] Fitzgibbon A, Pilu M and Fisher R B 1999 Direct least square fitting of ellipses leee Transactions on Pattern Analysis and Machine Intelligence 21 476-80

[14] Andersen N K and Taboryski R 2015 Multi-height structures in injection molded polymer Microelectronic Engineering 141 211-4

[15] Larsen S T, Andersen N K, Sogaard E and Taboryski R 2014 Structure Irregularity Impedes Drop RollOff at Superhydrophobic Surfaces Langmuir 30 5041-5

[16] Paxson A T and Varanasi K K 2013 Self-similarity of contact line depinning from textured surfaces Nature Communications 4

[17] Zimmermann J, Seeger S and Reifler F A 2009 Water Shedding Angle: A New Technique to Evaluate the Water-Repellent Properties of Superhydrophobic Surfaces Textile Research Journal 79 1565-70

[18] Courbin L, Denieul E, Dressaire E, Roper M, Ajdari A and Stone H A 2007 Imbibition by polygonal spreading on microdecorated surfaces Nature Materials 6 661-4 